\title{
Calorimetric Studies of Microbial Growth: Quantitative Relation between Growth Thermograms and Inoculum Size
}

\author{
Mamoru HaShimoto and Katsutada TAKAHASHI* \\ Laboratory of Biophysical Chemistry, College of Agriculture, \\ University of Osaka Prefecture, Sakai, Osaka 591, Japan
}

Received November 4, 1981

\begin{abstract}
Heat evolution during growth of bakery yeast was studied in a heat-conduction calorimeter. Growth thermograms observed for yeast grown on liquid synthetic media with various inoculum sizes were compared. The quantitative relation between actual heat evolution and inoculum size was discussed in terms of the exponential growth function.
\end{abstract}

Since pioneering work on the heat production during mold growth was performed by Tamiya and his coworkers in the $1930 \mathrm{~s},{ }^{1,2)}$ calorimetric investigations on microbial systems have been made by many workers. Furthermore; recent improvements in calorimetric techniques have enabled more quantitative application of calorimetry to many microbial systems and various kinds of investigation are actively being conducted in various fields. $^{3,4)}$

One of the possible applications of calorimetry to microbial systems is the kinetic analysis of growth thermograms that are obtained by continuous recording of the calorimeter signal during growing cultures. However, very few studies have been reported so far on the growth kinetics studied by calorimetric measurements. ${ }^{5,6)}$ In the present investigation, heat evolution during growing cultures of bakery yeast with different inoculum sizes was measured in a calorimeter and the quantitative relation between the growth thermogram and the inoculum size was studied in view of growth kinetics.

\section{MATERIALS AND METHODS}

Calorimeter. The calorimeter used was of the twin conduction type developed in this laboratory especially for the purpose of following the heat evolution of microbial systems. ${ }^{7)}$ The structure of the calorimeter and its detection mode are schematically shown in Fig. 1.

The calorimeter cell which acts as a culture vessel (A) equipped with a stirrer (B) and an inoculation tube (C) has a volume of $300 \mathrm{~cm}^{3}$ and can be autoclaved prior to the calorimetric operation by taking it out of the calorimeter heatsink (D, thermostated metal block). Between the cell and the heatsink, semiconducting thermopile plates $(\mathbf{E})$ are placed which serve as a thermal sensor. The calorimeter has two identical cells; a sample cell and a blank (dummy) cell. The differential voltage from the thermopile units of the two cells is taken as the calorimeter output signal and is recorded as a thermogram. Further details of the calorimeter and its operation were reported previously.7)

Bakery yeast. The bakery yeast used was Oriental Active Dry Yeast purchased from Oriental Yeast Co. Ltd., Tokyo, in the form of dry particles with viable yeast cells. ${ }^{* 1}$ The particle size is between $0.2 \mathrm{~mm}$ to $2 \mathrm{~mm}$ in diameter. The relative amount of viable cells can be most accurately and easily determined by weighing the particles.

The sample was stored in a refrigerator under nitrogen atmospheric pressure and was used as the seed for a culture by transferring the required amount of particles to the autoclaved culture vessel containing medium.

* To whom correspondence should be sent.

*1 This specimen contains at least two strains of yeast, Saccharomyces and Ellipsoidus. Since the specimen is a mixture of more than two strains, the growth thermogram represents the sum of the multiple microbial activities. Therefore, the thermograms observed in the present experiment correspond to the energetic activities of the whole system involving multiple microorganisms. The thermogram was reproducible when the specimens were cultured under the same conditions. 
Culture medium. Synthetic culture medium having the following composition was used: D-glucose, $800 \mathrm{mg}$; Lasparagine, $600 \mathrm{mg} ; \mathrm{KH}_{2} \mathrm{PO}_{4}, 200 \mathrm{mg} ; \mathrm{Na}_{2} \mathrm{HPO}_{4} \cdot 12 \mathrm{H}_{2} \mathrm{O}$, $200 \mathrm{mg} ; \mathrm{MgSO}_{4} \cdot 7 \mathrm{H}_{2} \mathrm{O}, 100 \mathrm{mg}$; yeast extract, $400 \mathrm{mg}$; $\mathrm{H}_{2} \mathrm{O}, 200 \mathrm{~cm}^{3} ; \mathrm{pH}, 5.7$.

Calorimetric operation. The culture vessel containing $200 \mathrm{~cm}^{3}$ of the medium was autoclaved and then placed in the calorimeter of which the heatsink was kept at $303 \mathrm{~K}$ by circulating thermostated water. The reference vessel containing $200 \mathrm{~cm}^{3}$ of water was also placed in the calorimeter. The medium in the culture vessel and the water in the reference vessel were stirred at a constant rate of 450 rpm. Under these conditions the dissolution rate constant

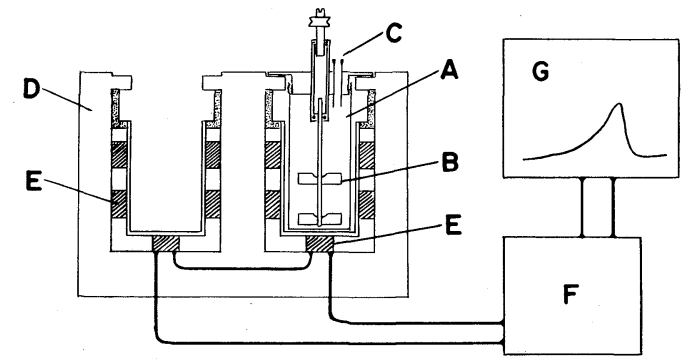

FIG. 1. Schematic Representation of the Calorimeter Structure and Its Measuring Mode.

(A) culture vessel; (B) stirrer; (C) inoculation tube; (D) heatsink; (E) thermopile plate; (F) DC-amplifier; (G) recorder. Details are given in ref. 7. of air-oxygen into the medium was found to be 0.070 $\min ^{-1} .^{* 2}$ After thermal equilibrium had been attained as judged from the recording of the calorimeter signal, the accurately weighed particles of dry yeast were transferred into the vessel through the inoculation tube in the lid of the vessel. The inoculum sizes were varied from $1.20 \mathrm{mg}$ to $20.20 \mathrm{mg}$ by weight. The growth thermograms were continuously recorded for 10 to 15 hours.

\section{RESULTS AND DISCUSSION}

Figure 2 shows the thermograms obtained for growth cultures with six different inoculum sizes. It is obvious that all the thermograms are essentially the same with respect to their shape; only the time scale is shifted to the left as the inoculum size is increased. In Fig. 3 one of the thermograms (with the inoculum size $N_{0}=$ $1.20 \mathrm{mg}$, solid line) is given together with the change in turbidity of the medium (triangles). The turbidity measurement was made on a separate growing culture under the same conditions as those employed for the calorimetric run. The relation between the thermogram and the turbidity change is quite similar to that previously observed for a pure culture of Sacch. cerevisiae. ${ }^{7)}$

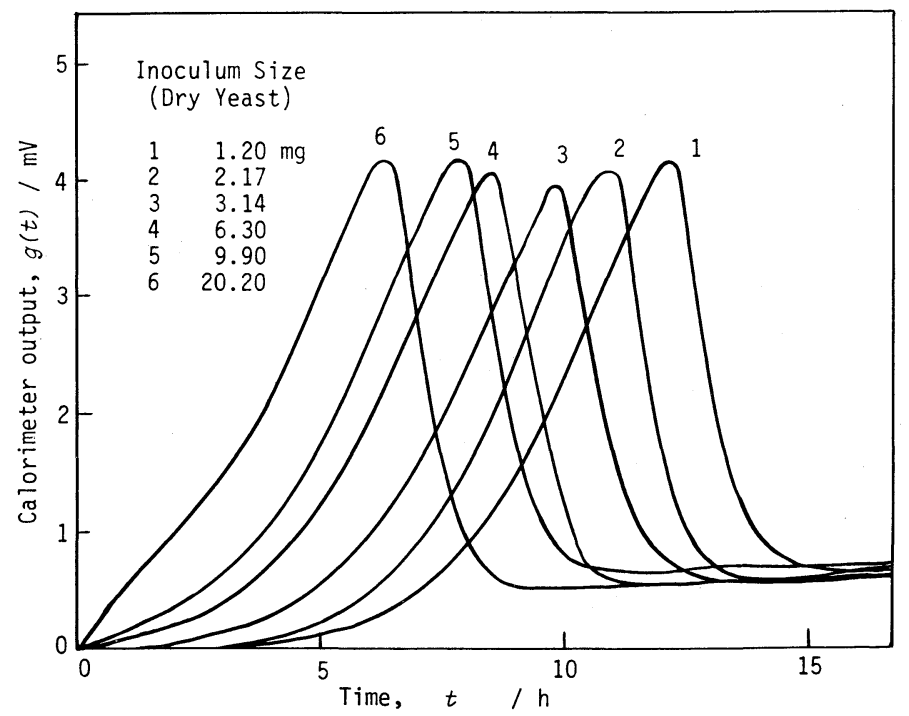

FIG. 2. Growth Thermograms Observed for Cultures with Various Inoculum Sizes.

*2 The rate of oxygen dissolution was determined by a separate measurement using an oxygen electrode. The rate constant is expressed as an apparent first-order rate constant obtained from the semi-log plot of the electrode signal versus time. 


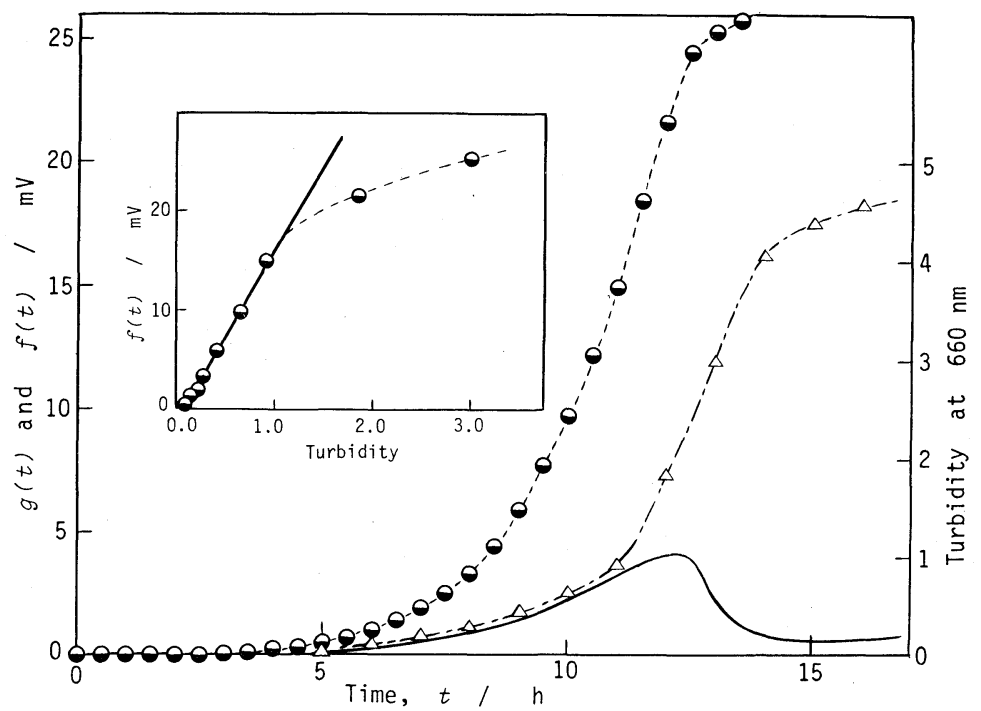

FIG. 3. Time Courses of Heat Evolution $f(t)(-\odot-)$ and Turbidity Change $\left(--\triangle^{--}\right)$.

$f(t)$ was calculated from the calorimeter signal $g(t)(-)$ by eq. (1). The inset shows the relation between the heat evolution and the turbidity change.

The observed thermogram is only an apparent calorimeter output signal and the true heat evolution in the calorimeter-cell is obtained when the thermogram is corrected for the heat exchange between the calorimeter cell and its surroundings through the calorimeter cell wall.

If we define $g(t)$, the calorimeter output signal at time $t$, the true heat evolution $f(t)$ is given by the following equation ${ }^{8,9}$;

$$
f(t)=g(t)+K \int g(t) d t
$$

where $K$ is the heat leakage modulus of the system. ${ }^{* 3} f(t)$ can be computed by a simple mathematical calculation ${ }^{8)}$ or more conveniently with a programable calculator. ${ }^{9)}$ In Fig. 3, $f(t)$ computed by eq. (1) is also included for comparison (half-closed circles). As for the heat leakage modulus, a value of $K=0.024$ $\min ^{-1}$, which was determined by using the Newton's heat exchange law, ${ }^{8,9)}$ was used. The calculation of $f(t)$ was done at time interval of $0.5 \mathrm{hr}$ with a Seiko S-301 Microcomputer.

$f(t)$ thus obtained is the time course of the calorimeter signal that one would observe when the system is hypothetically in an adiabatic condition and corresponds to the true process of heat evolution in the calorimeter cell. From Fig. 3, it can be seen that the true heat evolution process is quite similar to the change in turbidity.

The relation between the two became more clear when $f(t)$ was plotted against the turbidity change (the inset of Fig. 3). It appears that a good linear relationship exists between the heat evolution and the turbidity in the range up to an optical density of 1.0. This indicates that in the present microbial system the time course of heat evolution, $f(t)$, can also be regarded, to a certain extent, as a growth curve which is conventionally obtained by turbidity measurement.

On the basis of the above results, a theoretical consideration was made for the thermograms observed for the cultures with different inoculum sizes. $f(t)$ curves corresponding to the six thermograms in Fig. 2 were computed and are shown in Fig. 4. The number of cells in a growing culture of microbial cells is generally 


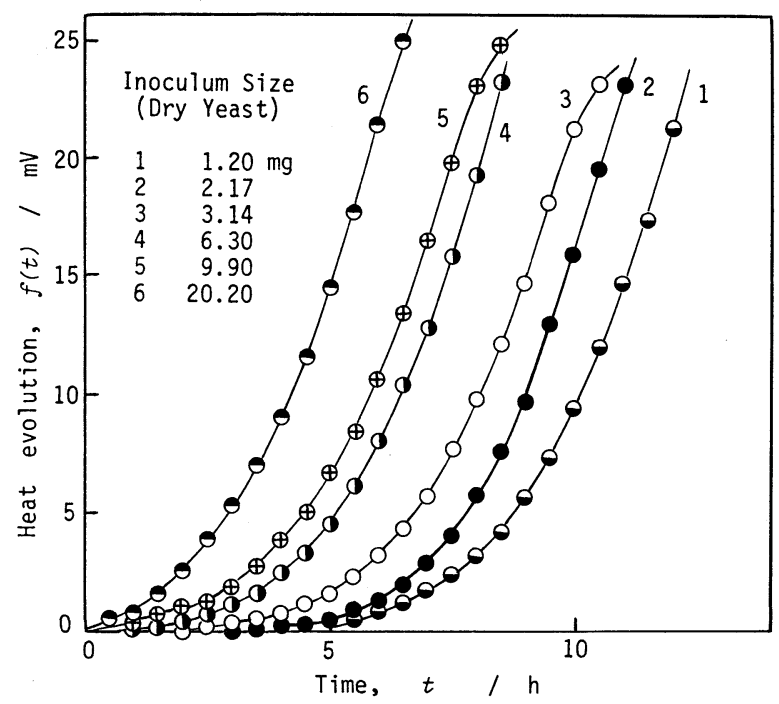

Fig. 4. Time Course of Heat Evolution Computed for Cultures with Six Different Inoculum Sizes.

expressed by the following equation;

$$
N=N_{0} \mathrm{e}^{\mu(t-\tau)}
$$

where $N$ is the number of viable cell at time $t$, $N_{0}$ is the inoculum size which is equal to the number of viable cells at $t=0, \mu$ is the growth rate constant and $\tau$ is the lag time.

If we define $q_{0}$ as heat evolution rate per unit viable cell during the lag phase and $q_{1}$ as heat evolution rate per unit cell during the exponential growth phase, the heat evolutions of the system during the lag and the exponential growth phases are given by

$$
\int_{0}^{\tau} N_{0} q_{0} \mathrm{~d} t \quad \text { and } \quad \int_{\tau}^{t} N q_{1} \mathrm{~d} t
$$

respectively.

The sum of the above two terms is the integral heat evolution at time $t(t>\tau)$. Thus we obtain;

$$
f(t)=\int_{0}^{\tau} N_{0} q_{0} \mathrm{~d} t+\int_{\tau}^{t} N q_{1} \mathrm{~d} t
$$

Equation (4) is a strict mathematical expression of the heat evolution during the exponential growth of microbial cells.

Substituting eq. (2) to eq. (4), we obtain

$$
f(t)=\int_{0}^{\tau} N_{0} q_{0} \mathrm{~d} t+\int_{\tau}^{t} q_{1} N_{0} \mathrm{e}^{\mu(t-\tau)} \mathrm{d} t
$$

Since, by definition, the heat evolution rate per unit cell during the exponential growth phase, $q_{1}$, is constant, eq. (5) can be rewritten as

$$
f(t)=N_{0} A \mathrm{e}^{\mu t}+N_{0} B
$$

where

$$
A=\frac{q_{1}}{\mu \mathrm{e}^{\mu \tau}} \quad \text { and } \quad B=\int_{0}^{\tau} q_{0} \mathrm{~d} t-\frac{q_{1}}{\mu}
$$

Thus $f(t)$ is given as an exponential function, from which the growth rate constant, $\mu$, is obtained by the Guggenheim method. ${ }^{9,10}$ )

The Guggenheim equation is derived from eq. (6) as

$$
\begin{aligned}
& \log \{f(t+\Delta t)-f(t)\} \\
= & \log N_{0} A\left(\mathrm{e}^{\mu \Delta t}-1\right)+\frac{\mu}{2.303} t
\end{aligned}
$$

where $\Delta t$ is an appropriate time interval.

Plotting $\log \{f(t+\Delta t)-f(t)\}$ against $t$, a straight line is obtained, the slope of which is $\mu / 2.303$.

In Fig. 5, the Guggenheim plots obtained for the six calorimetric runs are shown. It can be seen that all the plots are linear with nearly the same slope. The values of the growth rate constant, $\mu$, determined for each plot by the least square method are summarized in Table 
I. The average value obtained was $\mu=0.4982$ $\mathrm{h}^{-1}$ with a standard deviation of \pm 0.0578 .

The fact that the growth rate constants obtained for the different calorimetric runs agree well with each other suggests that the growth thermogram may be expressed as the same exponential function only with $N_{0}$ as a variable. Thus the results shown in Table I seem to support the validity of eq. (6).

In order to verify this, the relation between

Table I. Growth Rate Constant DETERMINED FROM THE GUGGENHEIM Plot

\begin{tabular}{ccc}
\hline $\begin{array}{c}\text { Calorimetric } \\
\text { run }\end{array}$ & $\begin{array}{c}\text { Inoculum size } \\
\left.\text { (dry yeast } N_{0} / \mathrm{mg}\right)\end{array}$ & $\begin{array}{c}\text { Growth rate } \\
\text { constant } \\
\left(\mu / \mathrm{h}^{-1}\right)\end{array}$ \\
\hline 1 & 1.20 & 0.5262 \\
2 & 2.17 & 0.5797 \\
3 & 3.14 & 0.5087 \\
4 & 6.30 & 0.5042 \\
5 & 9.90 & 0.4589 \\
6 & 20.20 & 0.4112 \\
Mean & \multicolumn{2}{c}{$\mu=0.4982 \pm 0.0578^{*}$} \\
\hline
\end{tabular}

* Standard deviation. the inoculum size and the thermogram was more quantitatively analyzed. For given values of $\log \{f(t+\Delta t)-f(t)\}=\alpha$ and $t=t_{\alpha}$, eq. (7) is rewritten as

$$
t_{\alpha}=-\frac{2.303}{\mu} \log N_{0}+\frac{2.303}{\mu}\left\{\alpha-\log A\left(\mathrm{e}^{\mu \Delta t}-1\right)\right\}
$$

Thus, the time required to give a definite value of $\log \{f(t+\Delta t)-f(t)\}=\alpha$ is expressed as a function of $N_{0}$ and a plot of $t_{\alpha}$ against the logarithm of inoculum size $\log N_{0}$ should give a straight line with a slope of $-2.303 / \mu$.

The values of $t_{\alpha}$ at $\alpha=0.35$ determined by the least square method are given in Fig. 5. Since the Guggenheim plots show a linear relation in a range of $0<\log \{f(t+\Delta t)-$ $f(t)\}<0.70$, the middle point, 0.35 , was chosen as a definite value of $\log \{f(t+\Delta t)-$ $f(t)\}$.

In Fig. 6, the plot of $t_{\alpha}$ versus $\log N_{0}$ is shown. It is obvious that the plot gives a straight line and that the relation described by eq. (8) holds. From the slope of this straighi line, $\mu$ was determined to be $0.4498 \mathrm{~h}^{-1}$.

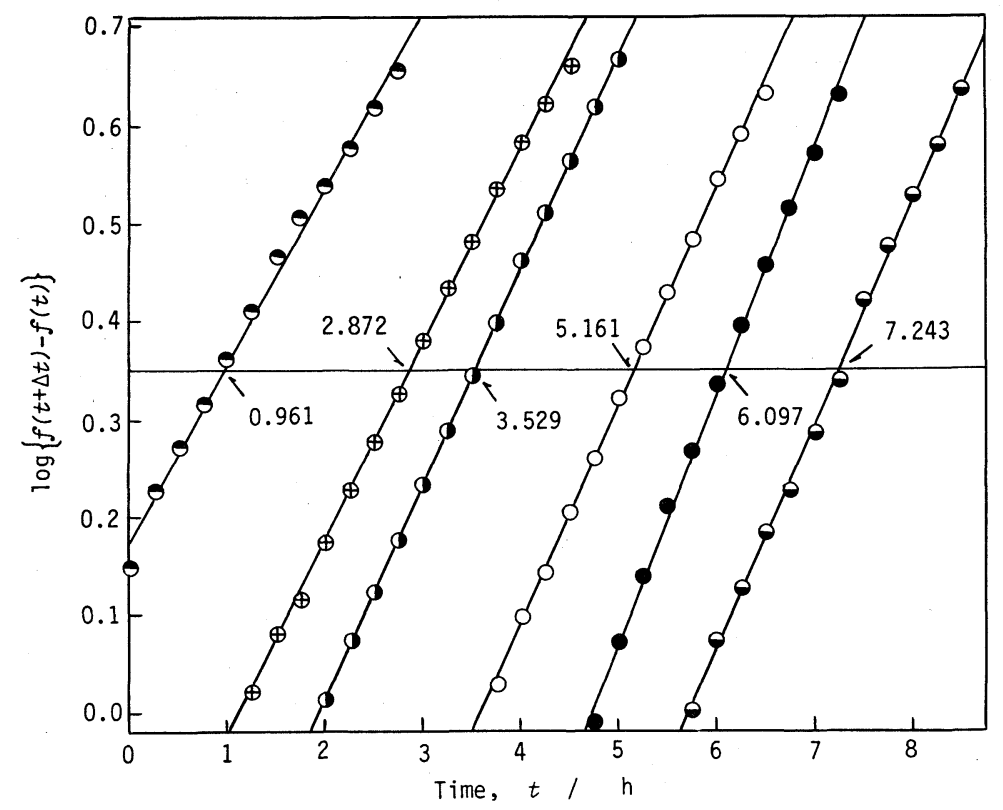

FIG. 5. Guggenheim Plots of the Heat Evolution Process during Growth.

Symbols are the same as those in Fig. 4. The number at each arrow indicates the intercept on $\log \{f(t+\Delta t)-f(t)\}=0.35$. 


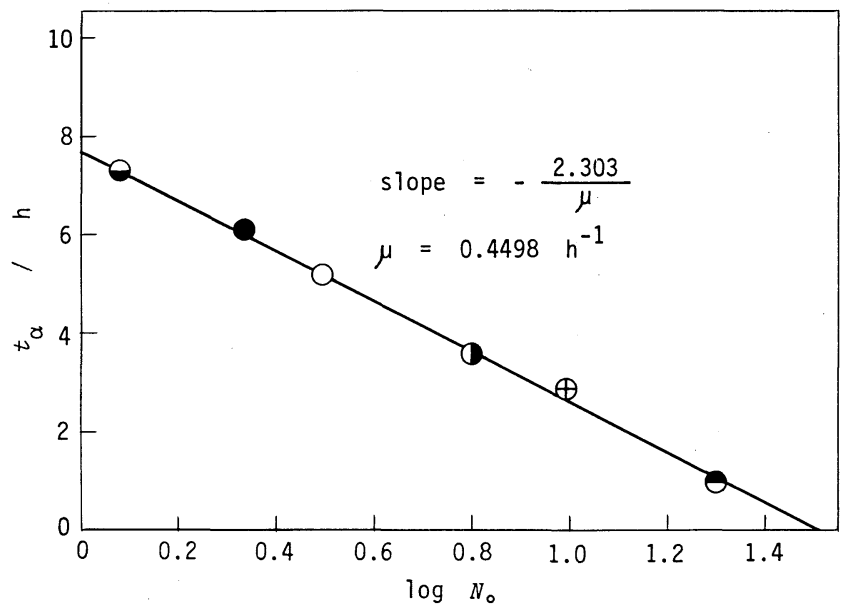

FIG. 6. Plot of $t_{\alpha} v s . \log N_{0}$.

Symbols are the same as those in Fig. 4.

The values determined independently for each calorimetric run (Table I) agree with the present value within the experimental error. Thus eq. (6) was proved to be valid for describing the heat evolution during the exponential growth.

\section{REFERENCES}

1) H. Tamiya and A. Yamamoto, Acta Phytochimica, 7, 245 (1933).

2) A. Yamamoto and H. Tamiya, Acta Phytochimica, 8, 245 (1935).

3) T. Fujita and K. Nonomura, "Application of Calorimetry in Life Sciences," ed. by I. Lamprecht and B. Schaarschmidt, Walter de Gruyter, Berlin and New York, 1977, p. 119; A. E. Beezer, ibid., p. 109; R. Brettel, ibid., p. 129; B. Schaarschmidt, A. I. Zotin and I. Lamprecht, ibid., p. 139; P. Monk, W. Forrest and I. Wadsö, ibid., p. 149 (including additional references).

4) J. P. Belaich, "Biological Microcalorimetry," ed. by A. E. Beezer, Academic Press, London, New York, Toronto, Sydney and San Francisco, 1980, p. 1; I. Lamprecht, ibid., p. 43; R. D. Newell, ibid., p. 163 (including additional references).

5) J. P. Belaich, J. C. Senez and M. Murgier, J. Bacteriol., 95, 1750 (1968).

6) M. Murgier and J. P. Belaich, J. Bacteriol., 105, 573 (1971).

7) K. Takahashi, Agric. Biol. Chem., 37, 2743 (1973).

8) S. Ono, K. Hiromi and K. Takahashi, J. Biochem., 57, 799 (1965).

9) K. Takahashi, "Seikagaku Jikken Koza," Vol. 12, ed. by The Japanese Biochemical Society, Tokyo Kagaku Dojin, Tokyo, 1976, p. 19.

10) E. A. Guggenheim, Philos. Mag., 2, 538 (1926). 